\title{
Diacronie
}

Studi di Storia Contemporanea

$N^{\circ} 12,4 \mid 2012$

Sulle tracce delle idee

\section{Adriano Roccucci, Stalin e il patriarca. La Chiesa ortodossa e il potere sovietico}

\section{Lorenzo Ettorre}

\section{(2) OpenEdition}

Journals

Edizione digitale

URL: http://journals.openedition.org/diacronie/2432

DOI: 10.4000/diacronie.2432

ISSN: 2038-0925

Editore

Association culturelle Diacronie

Notizia bibliografica digitale

Lorenzo Ettorre, "Adriano Roccucci, Stalin e il patriarca. La Chiesa ortodossa e il potere sovietico », Diacronie [Online], N 12, 4 | 2012, documento 14, online dal 29 décembre 2012, consultato il 10 décembre 2020. URL : http://journals.openedition.org/diacronie/2432 ; DOI : https://doi.org/10.4000/ diacronie. 2432 


\section{Diacronie}

\section{RECENSIONE:}

\section{Adriano ROCCUCCI, Stalin e il patriarca. La Chiesa ortodossa e il potere sovietico, Torino, Einaudi, 2011, 509 pp.}

a cura di Lorenzo ETTORRE *

Se si eccettuano alcuni prodotti di elevato spessore storiografico ${ }^{1}$, in Italia gli studi sulle persecuzioni religiose in URSS appaiono modesti. Il palcoscenico è dominato da una pubblicistica di carattere giornalistico, spesso non avulsa da trite polemiche e redditizie provocazioni. Attraverso un'analisi certosina delle fonti al momento disponibili, invece, lo studio di Adriano Roccucci - uno dei massimi esperti dei rapporti tra Chiesa ortodossa e potere sovietico - si situa in una prospettiva decisamente diversa, dove il rigore dello storico si fonde con una mole impressionante di documentazione, edita ed inedita. Il risultato è un saggio di valore, nel quale si ripercorrono le diverse e travagliate fasi dei rapporti tra comunismo e Chiesa ortodossa, focalizzando l'attenzione sui più significativi cambiamenti di rotta che le mutevoli necessità storiche resero necessario attuare.

In una prima fase di questo processo - dalla rivoluzione del ' 17 al coinvolgimento dell'URSS nella guerra mondiale - il principale motivo ispiratore della politica religiosa sovietica era l'eliminazione della presenza dei credenti nella società comunista. Nel programma del partito, approvato nel marzo del 1919 dall'VIII ${ }^{\circ}$ congresso, che dedicò notevole attenzione alla questione religiosa, si affermava:

Per quello che riguarda la religione il partito comunista russo non è soddisfatto del solo decreto di separazione della chiesa dallo stato e della scuola dalla chiesa, cioè di quei provvedimenti che la democrazia borghese propone nei suoi programmi. Il

\footnotetext{
${ }^{1} \mathrm{Ci}$ si riferisce in particolare ai lavori di MOROZZO DELLA ROCCA, Roberto, Le Chiese ortodosse. Una storia contemporanea, Roma, Studium, 1997; VALENTE, Massimiliano (a cura di), Santa Sede e Russia da Leone XIII a Pio XI, Roma, Libreria Editrice Vaticana, 2006; RICCARDI, Andrea, Il secolo del martirio. I cristiani del Novecento, Milano, Mondadori, 2009.
} 
fine della politica bolscevica in questo campo è di favorire la completa sparizione dei pregiudizi religiosi² ${ }^{2}$

L'ostilità nei confronti della Chiesa era di natura ben più profonda di un'avversione di tipo politico. La Chiesa non era soltanto un'antagonista in un frangente, anche drammatico, dello scontro per la conquista del potere, ma anche un nemico di classe con cui il partito comunista doveva condurre una lotta risoluta, da cui non desistere, se non temporaneamente, per esigenze di tipo tattico. Per gli ideologi comunisti il carattere controrivoluzionario, di cui fu accusata la Chiesa nei primi anni dopo il 1917, non era un tratto congiunturale, ma un elemento strutturale, che aveva i suoi fondamenti nella sua visione del mondo e nella lotta di classe, principio «metafisico» dell'ideologia marxista-leninista. Nel disegno di costruzione della società comunista l'esistenza di un'organizzazione religiosa fondata su una visione del mondo irriducibile alla cornice dottrinaria onnicomprensiva del marxismo-leninismo costituiva una contraddizione evidente. La Chiesa ortodossa e le altre comunità religiose erano le uniche realtà sociali non omologabili ai principi dell'ideologia marxista-leninista presenti in Unione Sovietica. Ha scritto Roccucci:

\begin{abstract}
È esistita un'alterità irriducibile della Chiesa al sistema sovietico la cui manifestazione visibile è stata la celebrazione della liturgia, che, anche nei momenti di maggior coercizione da parte del regime, ha costituito uno spazio di diversità all'interno del mondo sovietico. [...] Lo Stato sovietico, quindi, non poteva avere una posizione neutrale nei confronti del fattore religioso, anzi la sua avversione alla Chiesa aveva, oltre a ragioni di tipo politico e storico, una chiara motivazione antireligiosa 3 .
\end{abstract}

Sulla scia delle feroci oppressioni anticontadine - nell'offensiva contro la presenza ortodossa nelle campagne le motivazioni antireligiose si univano alle ragioni della lotta contro la cultura contadina e agli obiettivi della battaglia per la collettivizzazione - e, in seguito, delle purghe staliniane degli anni Trenta, la persecuzione nei confronti della religione subì un'accelerazione senza precedenti in cui caddero vittime, indiscriminatamente, migliaia tra vescovi, esponenti del clero e semplici fedeli. Si accentuò, al contempo, la lotta ai simboli e alle icone religiose, che fin dal 1917 non aveva cessato di provocare distruzioni e saccheggi in ogni angolo del Paese.

\footnotetext{
${ }^{2}$ Cit. in ROCCUCCI, Adriano, Stalin e il patriarca. La Chiesa ortodossa e il potere sovietico, Torino, Einaudi, 2011, p. 41.

3 Ibidem, p. 42.
} 
Tuttavia, come ha scritto Graziosi, «se la Chiesa era stata vinta, il sentimento religioso non era stato affatto estirpato»4. Malgrado le pressioni del regime, infatti, il $56,7 \%$ della popolazione si dichiarò ancora religioso, spingendo il potere sovietico prima a intensificare la repressioni e poi, nel momento del pericolo, a fare appello anche a questi sentimenti per salvare se stesso 5 .

In effetti, l'imminenza della "guerra inevitabile» 6 e l'esistenza imprevista di una intensa vita ecclesiale clandestina, contribuirono ad indurre Stalin a rinunciare, quantomeno temporaneamente, ai piani di eliminazione di ciò che restava del patriarcato di Mosca e dell'intera ortodossia. Da un lato, infatti, un eventuale arresto del patriarca Sergij avrebbe provocato la reazione delle altre Chiese cristiane e conseguentemente delle opinioni pubbliche soprattutto dell'Europa occidentale, condizionando l'atteggiamento dei rispettivi governi nei confronti dell'URSS. Dall'altro, il permanere di una vivace vita ecclesiale clandestina, indusse a ritenere la presenza di una direzione ufficiale della Chiesa russa più controllabile e gestibile rispetto alla scivolosa galassia delle realtà illegali. Furono probabilmente questi fattori di politica internazionale a spingere Stalin, alla vigilia della seconda guerra mondiale, ad un cambiamento di indirizzo politico nel governo del paese. Ed è in questo quadro secondo Roccucci - «che si deve cercare una risposta alla domanda sul perché Stalin non abbia voluto procedere alla liquidazione completa della direzione ecclesiastica della Chiesa russa»7.

A ciò va aggiunto che gli avvenimenti del 1939-1940 avevano determinato un consistente allargamento dei confini della giurisdizione del patriarcato di Mosca. Erano diversi milioni i fedeli ortodossi che diventarono cittadini sovietici e membri della Chiesa russa, insieme a numerosi vescovi e preti. Questo ampliamento delle frontiere comportò per il patriarcato di Mosca un'inattesa disponibilità, seppur limitata, di strutture e di attori della vita ecclesiale. Comparvero di nuovo all'interno dei confini dell'URSS monasteri, istituti d'istruzione teologica, riviste religiose, istituzioni caritative, fraternità, biblioteche ecclesiastiche. Per la Chiesa russa si trattava di un'insperata iniezione di nuove energie, che alimentavano la speranza di rianimare la vita ecclesiale. Questa nuova situazione, come le precedenti, pur nel permanere di una condizione di grande incertezza per il futuro, lasciava sperare che il pericolo di una totale liquidazione della direzione ecclesiastica e delle strutture visibili della Chiesa si

\footnotetext{
4 GRAZIOSI, Andrea, L’Urss di Lenin e Stalin. Storia dell'Unione Sovietica, Bologna, Il Mulino, 2007, p. 381.

5 Cfr. i dati sono citati da Graziosi. Ibidem, p. 382.

${ }^{6}$ Cfr. PONS, Silvio, Stalin e la guerra inevitabile (1936-1941), Torino, Einaudi, 1995.

7 ROCCUCCI, Adriano, Stalin e il patriarca, cit., p. 118.
} 
fosse allontanato. In effetti, l'autore dà un significato non secondario a questo allargamento dei confini per la sopravvivenza della Chiesa ortodossa: a suo giudizio, «l'ortodossia, che come fattore identitario aveva una valenza piuttosto filorussa o comunque antipolacca, e la sottomissione delle strutture ecclesiastiche al patriarcato di Mosca, erano elementi che, almeno in questa fase, potevano favorire l'assimilazione, o quantomeno contrastare le spinte centrifughe» ${ }^{8}$.

L'apice di queste dinamiche - ed è la seconda fase nella suddivisione proposta da Roccucci - fu rappresentato dalla guerra, con la fondamentale funzione "patriottica" che la Chiesa russa aveva svolto in un momento di massima difficoltà per l'intera nazione, quando la follia delle armate hitleriane aveva reso, agli occhi dei popoli dell'est, la dominazione tedesca più invisa addirittura di quella sovietica. Il dittatore georgiano per motivare il suo popolo a battersi con tutte le forze contro l'invasore nazista, non fece leva sui valori del comunismo, ma su quelli della Russia. Il primo interlocutore divenne per lui la Chiesa ortodossa. Proprio per questo, superato il tornante più critico del conflitto, dopo le battaglie di Stalingrado e di Kursk, nella notte tra il 4 e il 5 settembre 1943 Stalin ricevette al Cremlino, nel suo studio, il metropolita di Mosca e Kolomna Sergij, - locum tenens del trono patriarcale -, il metropolita di Leningrado e Novgorod Aleksij e il metropolita di Kiev e Galic Nikolaj; tutto ciò avenne alla presenza di Molotov, del capo del NKGB Merkulov e del colonnello Georgij G. Karpov, responsabile del quinto dipartimento della seconda direzione del NKGB, nelle cui competenze rientravano il controllo e la repressione delle organizzazioni religiose. Roccucci individua in questo incontro il punto di cesura nei rapporti tra Chiesa e potere sovietico, giudicando l'avvenimento come un fatto non soltanto inedito ma anche prioritario per le sorti della sopravvivenza della Chiesa russa.

L'incontro era insolito, non tanto per l'orario notturno in cui si svolse - dalle 00.40 alle 2.00 - quanto per i protagonisti e il significato. Erano presenti da una parte le massime autorità dello Stato sovietico e dall'altra i vertici della Chiesa ortodossa. I disegni strategici sulla futura sistemazione dell'Europa e del mondo alla fine del conflitto costituivano in quei mesi uno dei temi prioritari all'attenzione della dirigenza sovietica, e di Stalin in particolare: l'aspetto religioso non venne sottovalutato nell'incontro - Stalin concesse in proposito l'autorizzazione alla convocazione di un concilio e all'elezione di un nuovo patriarca - ma l'interesse del leader sovietico era di coinvolgere la Chiesa russa nei suoi piani di espansione dell'influenza sovietica. In questi piani vanno ricercate le origini del colloquio, e in questo quadro "vanno comprese le domande di Stalin a Karpov sul patriarcato ecumenico e sugli altri

8 Ibidem, p. 132. 
patriarcati del Medio Oriente, sulle Chiese ortodosse di Jugoslavia, Bulgaria e Romania e sulla Chiesa russa all'estero»9.

Ma l'autore, ed è questo uno dei pregi del testo, pur entrando minuziosamente nei dettagli di ogni singola vicenda trattata non perde mai di vista il filo rosso della ricostruzione, riuscendo a dosare sapientemente vicende circostanziate e nodi tematici più generali ${ }^{10}$. È per questo che giudica necessario evidenziare che, pur nella relativa normalizzazione dei rapporti tra Stato e Chiesa dopo il settembre 1943,

l'orientamento dei vertici sovietici non era certo quello di tollerare un processo di riapertura delle chiese spontaneo e massiccio, quale quello avvenuto nei territori occupati dai tedeschi, né tantomeno di permettere una ripresa della vita ecclesiale tale da restituire alle strutture ecclesiastiche la visibilità, il radicamento e l’influenza nella società russa, che avevano precedentemente alla rivoluzione o anche nei primi anni di governo bolscevico ${ }^{11}$.

I dirigenti bolscevichi non potevano tollerare il consolidamento di un'istituzione che rappresentava potenzialmente un'alternativa al loro potere sulla società. Le fondamenta ideologiche del regime restavano immutate con tutti i loro postulati, compreso quello del definitivo sradicamento della religione dalla società comunista: l'«uomo nuovo» vagheggiato non avrebbe dovuto nutrire alcuna fede religiosa, né essa avrebbe dovuto occupare alcun posto nella società e nell'organizzazione dello Stato sovietico.

\footnotetext{
9 Ibidem, p. 174.

${ }^{10} \mathrm{Al}$ riguardo, è indicativo il rilievo sulla differenza di impostazione del potere sovietico dopo l'incontro del 1943 e la creazione di uno nuovo organismo statale atto a gestire i rapporti con la Chiesa. Scrive Roccucci: «È significativo il fatto che fosse indicata come funzione specifica del nuovo organismo statale proprio il rapporto diretto col patriarca e non con le strutture ecclesiastiche nel loro complesso. Da un punto di vista giuridico il patriarcato non aveva una sua personalità e la legislazione riconosceva solo l'esistenza delle associazioni religiose, cioè delle parrocchie. Si trattava di un capovolgimento della politica seguita negli anni Venti e Trenta, quando uno degli obiettivi principali delle autorità sovietiche era stato quello di favorire la nascita di scissioni all'interno della Chiesa, per indebolire fino alla disgregazione la direzione ecclesiastica e in particolare il patriarca». Cfr. Ibidem, p. 220.

${ }^{11}$ Ibidem, p. 203.
} 


\section{* L'autore}

Lorenzo Ettorre si è laureato in Storia presso l'Università Tor Vergata di Roma con una tesi sul Partito comunista italiano; ora è dottorando presso l'Università della Tuscia di Viterbo e cultore della materia in Storia contemporanea nello stesso ateneo. Dopo aver approfondito alcune tematiche relative ad aspetti specifici del colonialismo europeo in Africa («La Società antischiavista d'Italia (1888-1937)» in Studi Storici, 3/2012) il suo ambito di interesse attuale verte sull'analisi dei rapporti tra "mondo cattolico" e "mondo comunista" nell'Italia repubblicana.

URL: < http://www.studistorici.com/progett/autori/\#Ettorre >

\section{Per citare questo articolo:}

ETTORRE, Lorenzo, «Recensione: Adriano ROCCUCCl, Stalin e il patriarca. La Chiesa ortodossa e il potere sovietico, Torino, Einaudi, 2011, 509 pp.», Diacronie. Studi di Storia Contemporanea: Sulle tracce delle idee, 29/12/2012,

URL:<http://www.studistorici.com/2012/12/29/ettorre_numero_12/ >

Diacronie Studi di Storia Contemporanea $\}$ www.diacronie.it

Risorsa digitale indipendente a carattere storiografico. Uscita trimestrale.

redazione.diacronie@hotmail.it

Comitato di redazione: Marco Abram - Jacopo Bassi - Luca Bufarale - Gianluca Canè - Alessandro Cattunar - Alice De Rensis - Barbara Galimberti - Deborah Paci - Fausto Pietrancosta - Matteo Tomasoni - Luca Zuccolo

Diritti: gli articoli di Diacronie. Studi di Storia Contemporanea sono pubblicati sotto licenza Creative Commons 2.5

Possono essere riprodotti a patto di non modificarne i contenuti e di non usarli per fini commerciali. La citazione di

estratti è comunque sempre autorizzata, nei limiti previsti dalla legge. 